\title{
Small Modular Reactor Site Considerations for Uganda
}

\author{
Mukyala Kevin and Robert Murray Field \\ Department of Nuclear Power Plant Engineering, KEPCO International Nuclear Graduate School (KINGS), Ulsan 45014, Korea
}

Received: October 24, 2016 / Accepted: November 07, 2016 / Published: December 31, 2016.

\begin{abstract}
Uganda principally depends on hydropower for electricity generation. However, expansion of hydropower resources is limited, and with growing population and demand for electricity, the government of Uganda is considering integration of nuclear power into its energy mix. With the current and projected grid capacity, SMR (small modular reactor) technology is attractive since capacity can be added incrementally. This paper therefore presents results for SMR site selection in Uganda. Starting with twenty regional areas established by the Ugandan government, a site selection process is outlined which uses four levels of screening and ranking. First, exclusion criteria including seismicity, volcanic activity, water resources, and transportation distances were applied. This reduced the potential regional areas down to eight. Next, MAUT (multi-attribute utility theory) weighting was assigned to these eight areas for: (1) distance to large population centers, (2) ease of transportation, and (3) distance to seismically or volcanically active areas. From this ranking, four regional areas stood out and were then selected. These regions were then compared using various attributes including access to water and integration with the developing national grid. In the final step, the lead candidate region was subdivided into subzones to further refine the selection process.
\end{abstract}

Key words: SMR, site selection, site evaluation, reserve suitable area, MAUT, nuclear power plant.

\section{Introduction}

Uganda, a country with a population equal to that of Poland and a land area about that of the United Kingdom, is an East African country which straddles the equator. With its people largely engaged in agricultural, Uganda seeks to rapidly develop the electricity infrastructure to stimulate and expand its economy. Presently, electricity is largely supplied by hydropower and current energy projects are focused on expanding that resource. As hydropower becomes fully developed in combination with other domestic energy sources (e.g., geothermal power in the Western Rift Valley and bagasse) projections of future electricity supply from conventional sources do not meet forecasted demand.

In addition, Uganda is land-locked with no seaport access. This constrains the economic import of fossil fuel supplies such as coal and liquefied natural gas.

Corresponding author: Mukyala Kevin, B.Sc. physics/chemistry, graduate student, research field: small modular reactor site assessment.
With this limitation, nuclear power as a future energy resource comes under consideration.

The term SMR (small modular reactor) has been applied to two different but related concepts: small modular reactors, and small and medium reactors. Small refers to reactors with a design output of less than $300 \mathrm{MWe}$ while medium refers to reactors with outputs between 300 and 700 MWe.

However, here SMR signifies modular reactors which produce electrical output ranging from 50 to 180 MWe. The modular nature of the technology infers that the reactors can be factory built and shipped overland in one or two pieces for easy erection onsite $[1,2]$. The intent of the technology is to minimize commercial and technological risks, and reduce construction schedules.

SMRs considered for near term deployment include designs from Korea (system integrated modular advanced reactor, or "SMART"), Russia (SVBR-100), the U.S. (Holtec, mPower, NuScale, Westinghouse), and others. These have a power output between 50 and $225 \mathrm{MWe}$. For the SMR complex is to produce 1,000 
MWe, six to twelve SMRs are required to be located on $\sim 20$ hectares.

This type of reactor plant is appropriate for Uganda relative to limited domestic infrastructure and experience with large power plant projects. With a relatively small but developing grid, this technology is appropriate both in relation to the grid and to development of domestic support for a nuclear power program.

\section{Methodology}

Reported here is a site selection process for the determination of preferred locations for the first SMR complex in Uganda. SMR technology is scalable, with the expectation that any individual site would house many SMRs (e.g., from six to twelve), built and synchronized to the grid in a sequential fashion.

First, Fig. 1 illustrates the twenty regional areas as identified by the Uganda government ("reserve suitable areas" numbered RSA1 to RSA20 [3]). These areas range in size from $400 \mathrm{~km}^{2}(\mathrm{RSA} 1)$ to $\sim 40,000$ $\mathrm{km}^{2}$ (RSA9).

In Step 1, starting with a review of relevant literature on siting for NPPs (nuclear power plants) and SMRs $[4,5]$, a number of exclusion criteria were first applied to screen the RSAs based on expert judgment.

Next, in Step 2 avoidance criteria were applied using MAUT (multi-attribute utility theory) $[6,7]$ to

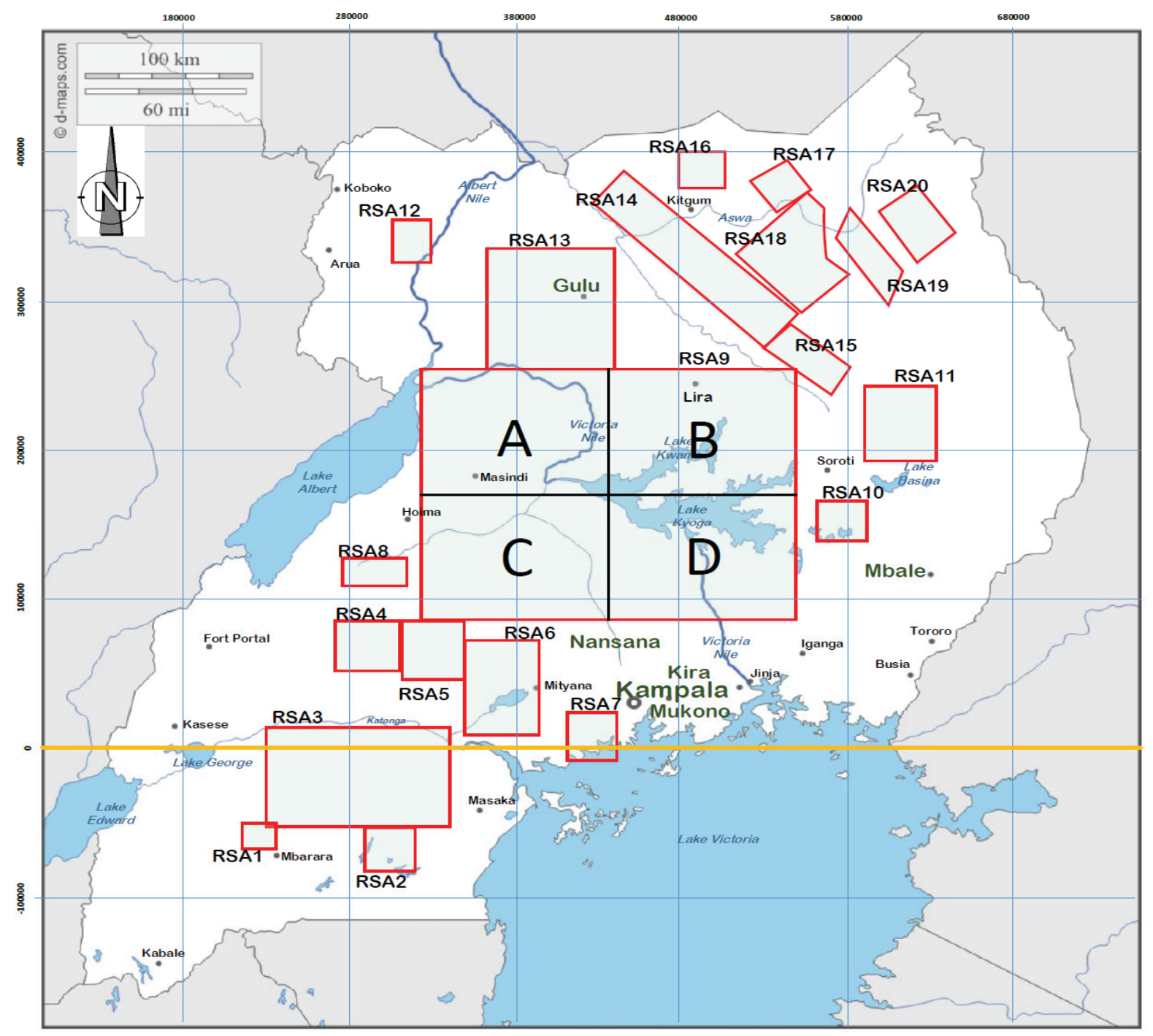

Fig. 1 Identified regional suitable areas. 
rank areas which passed the Step 1 screening to determine the order of relative preference. From this evaluation, preferred RSAs (top ranking) were selected.

In Step 3, using MAUT, suitability criteria were applied to the preferred regional areas from Step 2 screening to identify the lead candidate area.

Finally, in Step 4, four subzones within the lead candidate RSA were compared using MAUT methodology.

Details of the methods applied to the site selection and characterization process are described below.

\subsection{Step 1: Application of Exclusion Criteria}

Exclusion criteria were applied to eliminate less desirable RSAs. These criteria included seismicity, volcanic activity, water resources, transportation distances, and grid access.

For example, regions in the Western Rift Valley have a high incidence rate for seismic events. These areas also have a degree of volcanic activity which could present risk associated with ash fallout over a wide region. In addition, these areas experience landslides that can also be problematic.

Adequate water resources for some regions are not available outside of the rainy season. Some RSAs are located at distant locations relative to transportation and grid access.

\subsection{Step 2: RSA Avoidance Screening Using MAUT}

RSAs which were not eliminated in Step 1 were further analyzed using MAUT scoring for three categories: (i) proximity to the most populous cities, (ii) transportation network, and (iii) proximity to seismically active areas.

(1) Population-MAUT scoring for population was based on weighted distance to urban population centers. The largest cities in Uganda were identified and based on the national distribution of all city populations, the top six were selected (Kampala, Nansana, Kira, Mbarara, Mukono and Gulu) [8]. For each RSA, the distance to each major city was then combined with the city population to obtain an index indicating good separation. For a given RSA, this index was then totaled for all the six cities. The individual indices for the RSAs were then normalized to a value between 0 and 5 .

(2) Transportation-Major SMR equipment is expected to be delivered via ocean shipments through the seaport at Mombasa, Kenya. Therefore, the existing road network between Mombasa and each RSA was surveyed kilometer-by-kilometer using Google Earth imaging. Various impediments to transport of oversized heavy haul loads were tabulated. Impediments included: (i) travel through city centers including number of roundabouts, (ii) narrow road sections, (iii) underpasses, (iv) bridge crossings, (v) road surface type, and (vi) elevation changes. Based on a review of the tabulated impediments and distance to the border with Kenya, a subjective score between 0 and 5 was assigned to each RSA.

(3) Seismicity-Historically, seismic events have been recorded throughout Uganda. However, the most active and energetic events are stretched along the Western Rift Valley. Since there have been several hundreds of recorded seismic events of varying magnitudes, to make the MAUT scoring tractable, the locations of the six active volcanos which are interspersed within the valley were selected as a surrogate for seismically active locations to be avoided. Similar to MAUT scoring for population, distances between each RSA and each volcano were determined. Since seismic ground motion generally attenuates with the square of the distance from the epicenter, the sum of the reciprocals of the square of the distance to each volcano was computed for each RSA. The individual indices for all the RSAs were again normalized to a value between 0 and 5 .

Combined MAUT score - the normalized scores for population, transportation, and seismicity were then weighted and combined into a single score for each site. Final weighting for the three categories was 
selected by expert judgment as $20 \%, 40 \%$, and $40 \%$, respectively. The top four scoring RSAs were then selected for further review (Step 3). Note that other category weightings (e.g., 10\%, 30\%, and 60\%) were considered. These tended to result in the same rankings and selections for preferred RSAs.

\subsection{Step 3: Screening of Preferred RSAs}

Four high scoring RSAs were identified in Step 2 and further analyzed using the MAUT method. Attributes for these RSAs were examined to judge the availability of candidate sites within each region. Individual site suitability was based on a range of key attributes which were assigned to four categories: (i) health, safety, and security, (ii) engineering and cost, (iii) socio-economic, and (iv) environmental factors.

Within the categories, attributes were graded on a scale of 0 to 5 consisting of: (i) availability of exclusion zones with low population density, (ii) low seismic/volcano activity, (iii) accessibility to cooling water, (iv) road access, (v) grid access, (vi) separation from industrial areas, (vii) land use, (viii) availability of local workforce, (ix) proximity to wetlands, (x) susceptibility to flooding, and (xi) distance to national parks. Weights were assigned to each attribute and category and the scoring was combined into a single score to obtain the relative ranking of the RSAs.

\subsection{Step 4: Analysis of Lead Candidate RSA, (RSA9)}

From Step 3, the lead candidate RSA was determined to be RSA9. However, the land area for this region is very large spanning a wide range of topographies, watersheds, population zones, and so on. Therefore for the final evaluation of siting, this region was subdivided into four equal area subzones, namely A (northwest), B (northeast), C (southwest) and D (southeast) as shown in Fig. 1. The only attributes which were applied to these subzones were those which distinguished one zone from another. The subset of "key" attributes was taken as: (i) proximity to national parks, (ii) availability of adequate cooling water, (iii) avoidance of flooding, and (iv) access to the grid.

\section{Results and Discussion}

Uganda is generally well situated with minimal risk to SMRs associated with external events. Such events include seismicity, cyclones, tornados, tsunamis, high winds, icing, severe storms, and flooding $[9,10]$. A review of various sources confirms this.

For example, meteorological and flooding data [11, 12] for the entire country addressing extreme wind, precipitation, and flooding indicates low to moderate values. There is virtually no possibility of cyclone damage based on the landlocked position relative to the Indian Ocean.

As for tornados, recent experience indicates only a single event near Lake Victoria (2013). A review of the flooding history showed that only areas located in the north eastern part of Uganda were susceptible to recurring floods (see Fig. 2).

Therefore, with the exception of seismicity, external events are not considered to significantly differentiate one location from another within Uganda. The balance of this section provides analysis and results for the site selection process (as described in Section 2 above).

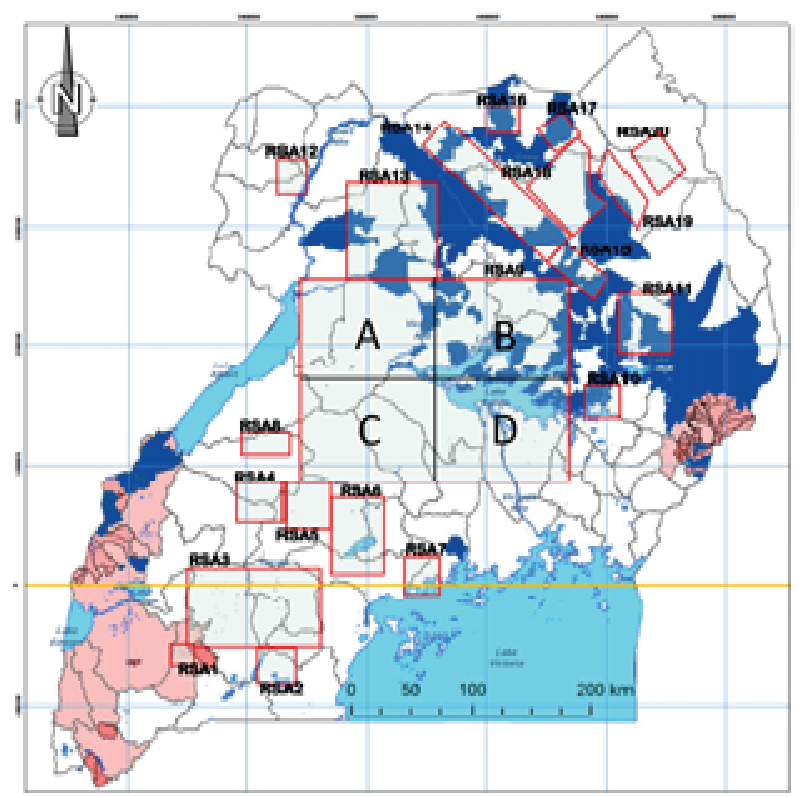

Fig. 2 Floods (blue) and landslides (brown) areas. 


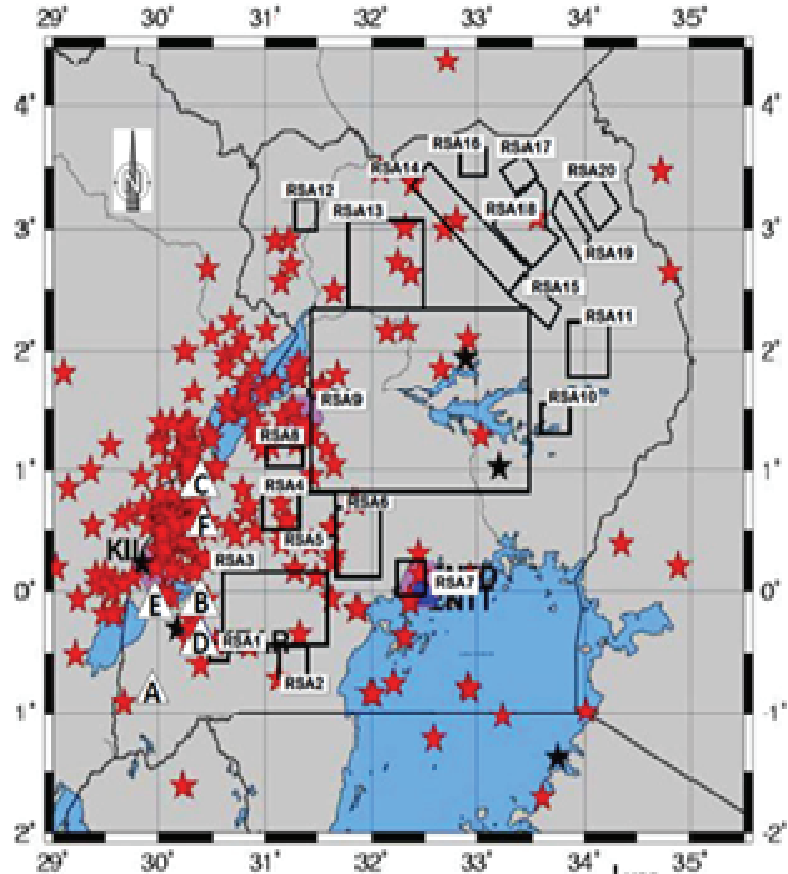

Fig. 3 Geological hazards in Uganda.

\subsection{Step 1: Application of Exclusion Criteria}

Using the exclusion criteria related to seismicity and volcanic activity, reserve suitable areas RSA1, RSA2, RSA3, RSA4, and RSA8 were screened out due to their location within regions of high seismicity and volcanic activity. Fig. 3 indicates recently recorded seismic events. Red stars show locations of earthquake epicenters for the period 2000 through 2009. Black stars indicate earthquakes which occurred in 2010. Triangles labeled $\mathrm{A}$ through $\mathrm{F}$ indicate active volcanoes (Bufumbira, Bunyaruguru, Fortportal, Mount Katunga, Katwe-kirongo and Kyatwa, respectively [13]).

Next, distance to transportation routes and grid accessibility were considered for the remaining areas (see Fig. 4). Seven regions, RSA12, RSA14, RSA16, RSA17, RSA18, RSA19 and RSA20 were found to be far from both transportation routes, and the current and planned transmission network and were screened out [14].

Using this process, twelve RSAs were eliminated from further consideration. The eight RSAs that qualified for further evaluation were: RSA5, RSA6, RSA7, RSA9, RSA10, RSA11, RSA13, and RSA15.

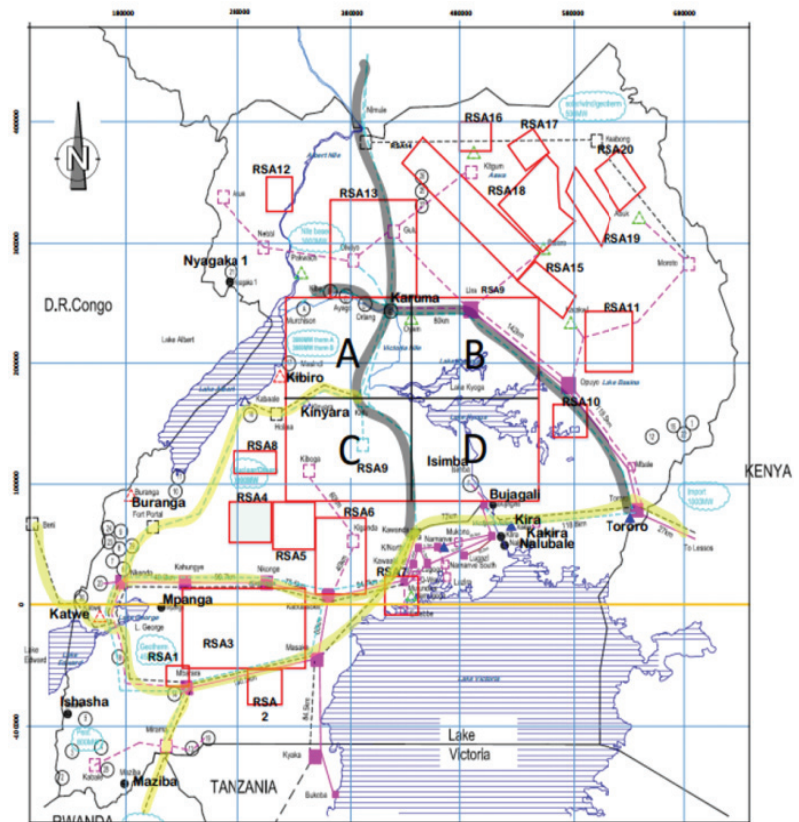

Fig. 4 Uganda electrical grid $(220 \mathrm{kV}$-yellow, 400 kV-gray).

Table 1 Step 2 MAUT scoring and ranking.

\begin{tabular}{llllll}
\hline Sites & Population & Transport & Seismic & $\mathrm{OA}^{\mathrm{a}}$ & $\mathrm{RR}^{\mathrm{b}}$ \\
\hline Weight & $20 \%$ & $40 \%$ & $40 \%$ & $100 \%$ & - \\
RSA5 & 2.96 & 1.00 & 1.32 & 1.6 & 8 \\
RSA6 & 2.60 & 3.00 & 0.68 & 2.0 & 6 \\
RSA7 & 1.61 & 4.00 & 2.03 & 2.8 & 3 \\
RSA9 & 3.13 & 3.00 & 1.96 & 2.6 & 4 \\
RSA10 & 2.65 & 5.00 & 4.54 & 4.4 & 1 \\
RSA11 & 4.09 & 3.50 & 4.57 & 4.1 & 2 \\
RSA13 & 0.93 & 2.00 & 1.87 & 1.7 & 7 \\
RSA15 & 4.69 & 1.00 & 1.84 & 2.1 & 5 \\
\hline
\end{tabular}

a) $\mathrm{OA}$ - Overall score. b) $\mathrm{RR}$ - Relative ranking.

\subsection{Step 2: RSA Screening Using MAUT}

Step 2 analysis provided in Table 1 indicates that areas RSA7, RSA9, RSA10, and RSA11 exhibit good regional characteristics. These include an adequate buffer to both earthquake prone areas and populated cities, and good access to developed overland transportation routes. Areas RSA5, RSA6, and RSA13 are near earthquake prone regions and/or close to populated cities. RSA15 is distant from major highways and is hence screened out. Thus RSA7, RSA9, RSA10, and RSA11 were selected as preferred regions to advance to Step 3 for further analysis. Weighting and scoring is provided in Table 1. 


\subsection{Step 3: Screening of Preferred RSAs}

The four preferred regions from Step 2 were next evaluated against the eleven criteria described in Section 2.3. Circumstances and features specific to each RSA which factored into the MAUT scoring are discussed below. Composite scoring is then provided in Table 2 . A summary description of the subcategory evaluations for the RSAs is provided below.

\subsubsection{Analysis for RSA7}

This region generally has high population density and it is difficult to locate suitable sites with low populations in the exclusion zone. On the other hand, this region has good access to local labour. The region has high seismicity relative to the other preferred regions. There is no history of severe flooding but much of the area is located within permanent wetlands. This RSA is far from national parks, and has sufficient access to cooling water and overland transport. Grid access is currently restricted to a nearby $220 \mathrm{kV}$ transmission line and would require an extension of the planned $400 \mathrm{kV}$ line. This region has relatively high current and future potential for industrial development and includes intensive land use associated with agriculture.

\subsubsection{Analysis for RSA9}

This region has many areas with low population density. It is relatively easy to locate suitable sites with populations of less than 15 persons per square kilometre in the exclusion zone (see Fig. 5). Beyond the exclusion zone, the current and projected population density is well below the guidance of 500 persons per square kilometre given in 10CFR100.11.

This RSA has low seismicity, adequate cooling water from both Lake Kyoga, and the Victoria Nile. Also it has relatively low land utilization with moderate levels of agriculture, and very good access to $400 \mathrm{kV}$ and $220 \mathrm{kV}$ transmission lines. The region is distant from industries, has minimal historical flooding, and is surrounded mainly by permanent and seasonal wet lands. Part of this RSA is near a national park and may be crossed by migratory species.

\subsubsection{Analysis for RSA10}

RSA10 has a high population density $(\sim 300$ persons per square kilometre). Therefore locating suitable sites within this region is problematic. Seismicity is considered to be low (high scoring). The local labour force is considered to be adequate. However, much of this region is located within permanent and seasonal wetlands. High seasonal flood

Table 2 Step 3 MAUT weighting of four reserve suitable areas.

\begin{tabular}{|c|c|c|c|c|c|c|c|c|c|}
\hline & & \multicolumn{4}{|c|}{ Raw score } & \multicolumn{4}{|c|}{ Weighted score } \\
\hline \multicolumn{2}{|c|}{ Category/subcategory weighting ( 0 to $100 \%$ ) } & RSA7 & RSA9 & RSA10 & RSA11 & RSA7 & RSA9 & RSA10 & RSA11 \\
\hline Health, safety, and security & $50 \%$ & & & & & & & & \\
\hline Population within exclusion zone & $70 \%$ & 2.0 & 4.0 & 0.5 & 3.0 & 14.0 & 28.0 & 3.5 & 21.0 \\
\hline Seismicity, volcanism & $30 \%$ & 1.5 & 4.5 & 5.0 & 5.0 & 4.5 & 13.5 & 15.0 & 15.0 \\
\hline Engineering and cost & $30 \%$ & & & & & & & & \\
\hline Access to water & $35 \%$ & 5.0 & 5.0 & 2.0 & 2.5 & 10.5 & 10.5 & 4.2 & 5.3 \\
\hline Access to roads & $30 \%$ & 3.5 & 4.5 & 2.5 & 5.0 & 6.3 & 8.1 & 4.5 & 9.0 \\
\hline Access to grid & $25 \%$ & 2.5 & 5.0 & 4.5 & 4.0 & 3.8 & 7.5 & 6.8 & 6.0 \\
\hline Proximity to intensive industry & $10 \%$ & 0.5 & 2.0 & 0.5 & 0.5 & 0.3 & 1.2 & 0.3 & 0.3 \\
\hline Socioeconomic & $15 \%$ & & & & & & & & \\
\hline Land use & $40 \%$ & 0.5 & 3.5 & 3.0 & 3.0 & 0.6 & 4.2 & 3.6 & 3.6 \\
\hline Local workforce & $60 \%$ & 4.0 & 1.5 & 4.0 & 0.5 & 7.2 & 2.7 & 7.2 & 0.9 \\
\hline Environmental factors & $5 \%$ & & & & & & & & \\
\hline Proximity to wetlands & $35 \%$ & 0.5 & 3.0 & 0.5 & 4.0 & 0.2 & 1.1 & 0.2 & 1.4 \\
\hline Flooding & $60 \%$ & 5.0 & 4.0 & 0.5 & 0.5 & 3.0 & 2.4 & 0.3 & 0.3 \\
\hline National parks & $5 \%$ & 2.5 & 1.0 & 2.5 & 2.5 & 0.1 & 0.1 & 0.1 & 0.1 \\
\hline Totalized score ( 0 to 100$)$ & & & & & & 50 & 79 & 46 & 63 \\
\hline
\end{tabular}




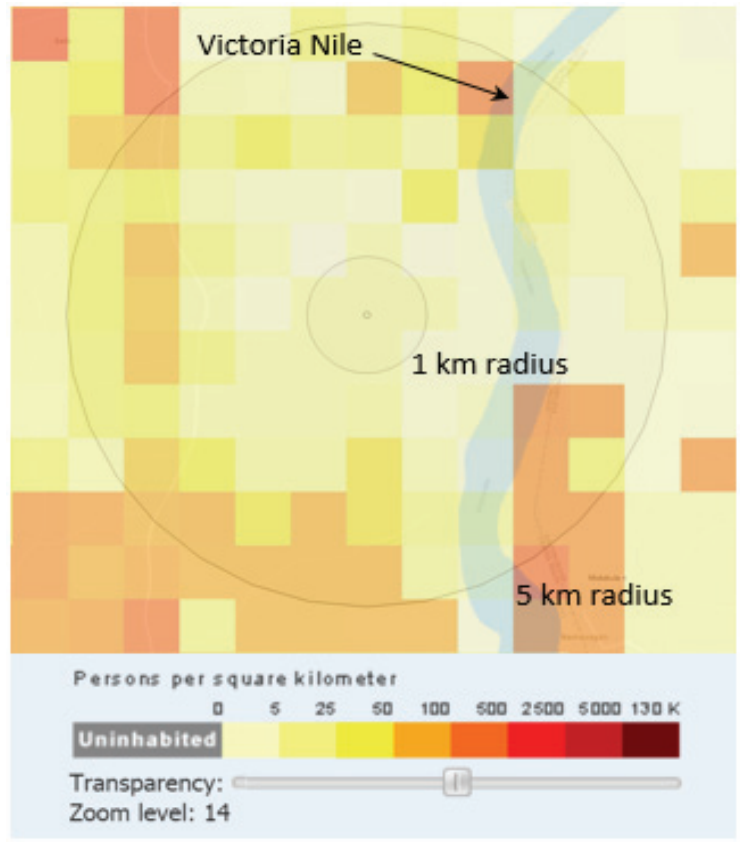

Fig. 5 RSA9 typical site population distribution.

levels can affect access to the site. There are limitations on water availability with possible cost implications associated with dry or hybrid cooling. Currently, developed road access is poor with a need to construct a road leading to the site. Interconnections to transmission lines of $220 \mathrm{kV}$, and $400 \mathrm{kV}$ can be easily effected (see Fig. 4). There is no expected interference with industrial facilities. Land use is poorly developed leaving many areas suitable for siting. This region is located far from national parks.

\subsubsection{Analysis for RSA11}

RSA11 has a relatively high population density. It is difficult to locate sites with exclusions zones containing less than 50 persons per square kilometre. Several areas within this region meet the recommendation to remain below 500 persons per square kilometre in the low population zone [15]. However, a rapidly growing population may be a challenge in the future. The local labour force is limited and not ideal. The region is not affected by seismicity, but has seasonal wetlands with high flood levels. Water for a year-round cooling is limited. There is good road access, and a planned nearby transmission line of $400 \mathrm{kV}$. However, a second offsite interconnection is lacking. The region is generally far from industries, has minimal land use, and is located far from national parks.

\subsection{Step 4: Analysis of Lead Candidate RSA}

Results in Table 2 indicate that RSA9 has the highest MAUT scoring and is then selected as the lead candidate regional area. RSA9 is also the largest of the twenty regions considered by the government study ( 40,000 square kilometers). To refine the analysis, this RSA was subdivided into four equal subzones, namely A, B, C and D (see Fig. 1). These subzones were then subjected to further MAUT screening using attributes for: (i) closeness to national parks, (ii) availability of year-round sources of cooling water, (iii) flooding, and (iv) current and projected grid access.

\subsubsection{Analysis of Subzone A}

There is a national park located entirely within the boundaries of subzone A. This will limit available locations for SMR sites. This subzone has excellent access to plentiful water supply from the Victoria Nile. Estimated consumptive water use for 1,000 MWe of installed SMRs using wet cooling towers is less than $1 \%$ of average annual river flow. There are no special limitations associated with flooding. Access to the planned route for $400 \mathrm{kV}$ transmission lines is good.

\subsubsection{Analysis of Subzone B}

There are no national parks located near this subzone. Water supply to this subzone is irregular and seasonal. Therefore, there will be an economic penalty for sites within this subzone to address cooling. Flooding is a significant issue for this subzone with an expectation of frequent and recurring seasonal floods. Grid access to the planned route for $400 \mathrm{kV}$ transmission lines is acceptable.

\subsubsection{Analysis of Subzone C}

There are no national parks located near this subzone. There is no identified source of water which can adequately service the plant. Therefore, dry or hybrid cooling will be required for any site within this subzone. Flooding is not a significant issue for this 
Table 3 Step 4 screening of subzones A, B, C, and D.

\begin{tabular}{llllll}
\hline & $\begin{array}{l}\text { Subcategory } \\
\text { weighting }\end{array}$ & \multicolumn{3}{c}{ Subzone } \\
\hline Subcategory & & $\mathrm{A}$ & $\mathrm{B}$ & $\mathrm{C}$ & $\mathrm{D}$ \\
\hline National park & $10 \%$ & 0.0 & 5.0 & 5.0 & 2.5 \\
Access to water & $40 \%$ & 4.0 & 2.5 & 1.0 & 5.0 \\
Flooding & $30 \%$ & 3.0 & 0.5 & 5.0 & 4.0 \\
Grid access & $20 \%$ & 5.0 & 4.0 & 4.0 & 3.5 \\
Final score & $(0$ to 100) & 70 & 49 & 64 & 83 \\
\hline
\end{tabular}

subzone. Grid access to the planned route for $400 \mathrm{kV}$ transmission lines is acceptable only for the eastern half of this subzone.

\subsubsection{Analysis for Subzone D}

There are no national parks located near this subzone. This subzone has excellent access to plentiful water supply from the Victoria Nile or Lake Kyoga. As was the case for subzone A, estimated consumptive water use is less than $1 \%$ of average annual river flow. There are no special limitations associated with flooding. There are no planned high voltage transmission corridors within this subzone (Fig. 3). However, projected costs for transmission lines are relatively low for Uganda. In addition, there is future potential for a hydropower project at Isimba, and interconnections between the SMR site and Isimba to Kampala and Tororo would complete the $400 \mathrm{kV}$ network into a national loop enhancing overall grid reliability.

Table 3 provides MAUT scoring for the subzones A, $\mathrm{B}, \mathrm{C}$, and $\mathrm{D}$.

\section{Conclusions and Recommendations}

Outlined here is a site selection process for determining the lead candidate areas for siting of an SMR complex in Uganda. The process made use of expert judgment and MAUT scoring. Candidate areas were taken as those selected in the Uganda government study. Twelve of the original twenty areas were first eliminated as low preference locales based on exclusionary criteria.

Next, avoidance criteria and MAUT scoring were applied to the eight remaining RSAs to further reduce the number of areas to only four. MAUT scoring was then applied using a set of suitability criteria to identify the lead candidate region.

Finally, the lead candidate region (RSA9) was divided into four subzones. Again MAUT scoring was used to identify the preferred subzone within this region. Note that this subzone $\mathrm{D}$, has a land area of $10,000 \mathrm{~km}^{2}$ whereas the site for an SMR complex is comprised of $\sim 20$ hectares for the site security boundary and $\sim 200$ hectares for the exclusion zone.

Determination of any particular lead candidate site for the SMR complex requires a local site specific study. Such a follow-on study would identify and examine individual sites in detail with regard to local land use, land ownership, public acceptance, topography, soil substructure, and other criteria related to site specific characteristics.

Note that while the siting study detailed here is identified with the introduction of an SMR complex with up to $\sim 1,000$ MWe capacity, the results are equally valid for the introduction of small to medium sized reactors with an equivalent capacity (e.g., $2 \times$ $600 \mathrm{MWe})$.

\section{Acknowledgments}

This research was supported by KINGS (KEPCO International Nuclear Graduate School), Republic of Korea and IAEA (International Atomic Energy Agency).

\section{References}

[1] Reichel, B. 2013. "The Role of Small Modular Reactors in Developing Countries." Accessed October 2, 2016. http://www.c-n-t-a.com/eventmain_files/zessay/13reichel. pdf.

[2] Ingersoll, D. T. 2009. "Deliberately Small Reactors and Second Nuclear Era." Progress in Nuclear Energy 51 (s 4-5): 589-603.

[3] Uganda Ministry of Energy and Mineral Development. Report on Site Survey for Nuclear Power Plants in Uganda, August 2015.

[4] IAEA (International Atomic Energy Agency). 2012. Managing Sitting Activities for Nuclear Power Plants. Nuclear Energy Series, Technical report No. NG-T-3.7, 
Vienna, Austria.

[5] IAEA (International Atomic Energy Agency). 2002. External Human Induced Events in Site Evaluation for Nuclear Power Plants. Safety Standards Series No. NS-G-3.1, Vienna, Austria.

[6] Baker, D., Bridges, D., Hunter, R., Johnson, G., Krupa, J., Murphy, J., and Sorenson, K. 2002. Guidebook to Decision Making Methods. WSRC-IM-2002-00002, Department of Energy, USA. Accessed October 2, 2016. http://academic.evergreen.edu/projects/bdei/documents/d ecisionmakingmethods.pdf.

[7] Papadopoulos, A. M., and Konidari, P. 2011. Overview and Selection of Multi-criteria Evaluation Methods for Mitigation/Adaptation Policy Instruments. National and Kapodistrian University of Athens Greece.

[8] UBOS. 2014. "National Population and Housing Census Provisional Results." Uganda Bureau of Statistics.

[9] Turner, K. 2002. Siting Guide: Site Selection and Evaluation Criteria for an Early Site Permit Application (Sitting Guide), EPRI-1006878.

[10] IAEA (International Atomic Energy Agency). 2011. Meteorological and Hydrological Hazards in Site
Evaluation for Nuclear Installation. Specific Safety Guide SSG-18, Vienna, Austria.

[11] Muwembe, K. Y. 2013. The Uganda Meteorological Services Data Observations, Management, Seasonal Forecasting and Early Warning System for Extremes. Uganda Department of Meteorology.

[12] Dartmouth Flood Observatory. Space-based Measurement of Surface Water. Dartmouth College. Accessed October 2, 2016. http://www.dartmouth.edu/ floods/Archives/GlobalArchi veMap.html.

[13] Siebert, L., and Simkin, T. 2002. "Volcanoes of the World: An Illustrated Catalog of Holocene Volcanoes and Their Eruptions." Smithsonian Institution, Global Volcanism Program Digital Information Series, GVP-3, http://www.volcano.si.edu.

[14] Uganda Electricity Transmission Co. Ltd. 2014. Grid Development Plant 2014-2030.

[15] Belles, R. J., and Omitaomu, O. A. 2014. Oak Ridge report on Population Sensitivity Evaluation of Two Candidate Locations for Possible Small Modular Reactor Siting Goals, Oak Ridge National Laboratory. 